Document downloaded from:

http://hdl.handle.net/10251/123290

This paper must be cited as:

Rodríguez-Älvarez, M.; Sánchez, F.; Soriano Asensi, A.; Moliner Martínez, L.; Sánchez Góez, S.; Benlloch Baviera, JM. (2018). QR-Factorization Algorithm for Computed Tomography (CT): Comparison With FDK and Conjugate Gradient (CG) Algorithms. IEEE Transactions on Radiation and Plasma Medical Sciences. 2(5):459-469.

https://doi.org/10.1109/TRPMS.2018.2843803

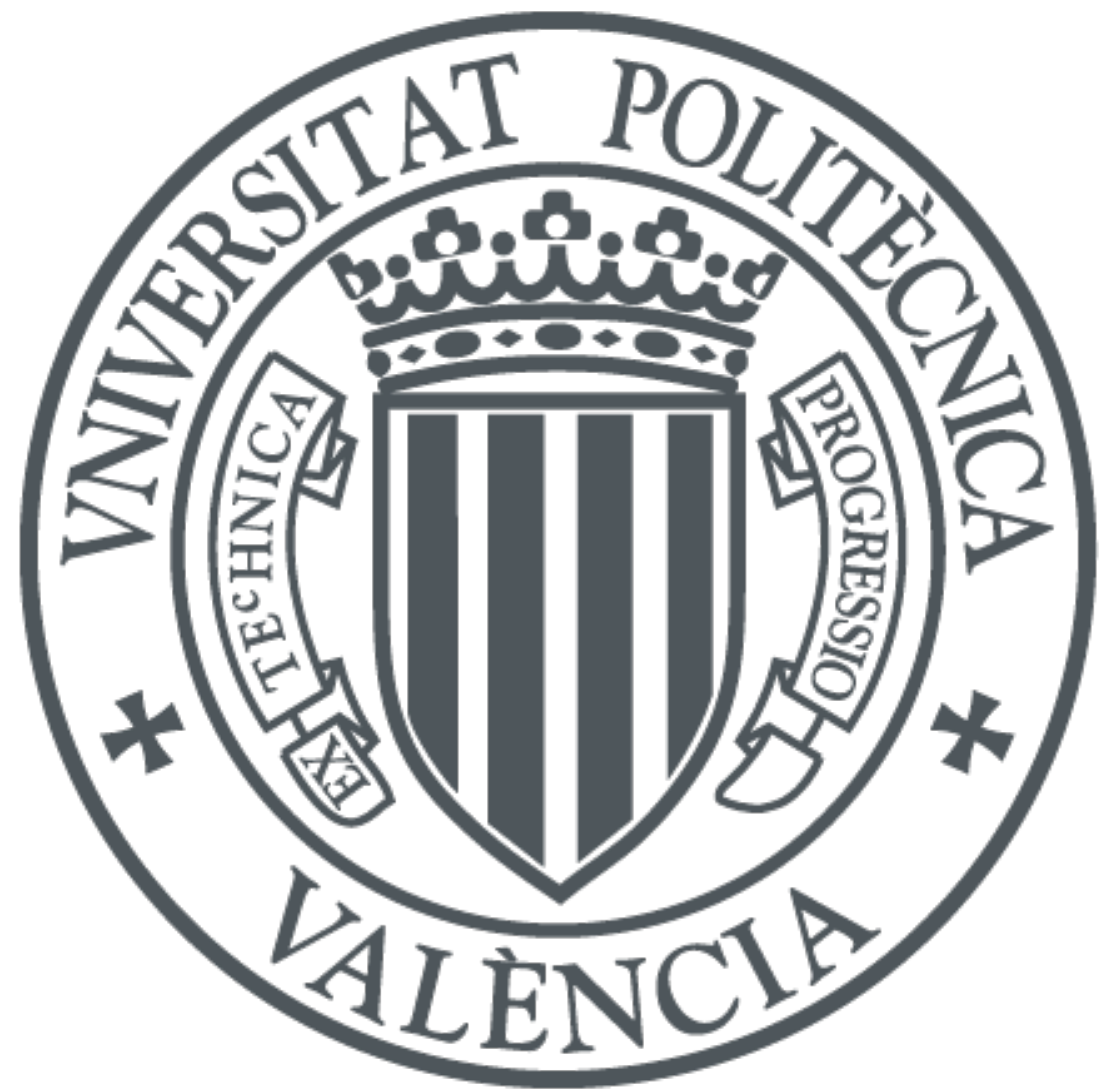

The final publication is available at

http://doi.org/10.1109/TRPMS.2018.2843803

Copyright Institute of Electrical and Electronics Engineers

Additional Information 


\title{
QR-factorization Algorithm for Computed Tomography (CT): Comparison with FDK and Conjugate Gradient (CG) Algorithms.
}

\author{
María José Rodríguez-Alvarez, Filomeno Sánchez, Antonio Soriano, Laura Moliner, Sebastián \\ Sánchez, José María Benlloch
}

\begin{abstract}
Even though QR-factorization of the system matrix for tomographic devices has been already used for medical imaging, to date, no satisfactory solution has been found for solving large linear systems, such as those used in Computed Tomography (CT) (in the order of $10^{6}$ equations).

In computed tomography, the Feldkamp, Davis and Kress back projection algorithm (FDK) and iterative methods like conjugate gradient (CG) are the standard methods used for image reconstruction. As the image reconstruction problem can be modelled by a large linear system of equations, QR-factorization of the system matrix could be used to solve this system. Current advances in computer science enable the use of direct methods for solving such a large linear system. The QR-factorization is a numerically stable direct method for solving linear systems of equations, which is beginning to emerge as an alternative to traditional methods, bringing together the best from traditional methods.

QR-factorization was chosen because the core of the algorithm, from the computational cost point of view, is pre-calculated and stored only once for a given CT system, and from then on, each image reconstruction only involves a backward substitution process and the product of a vector by a matrix.

Image quality assessment was performed comparing contrast to noise ratio (CNR) and noise power spectrum (NPS); performances regarding sharpness were evaluated by the reconstruction of small structures using data measured from a small animal 3D CT.

Comparisons of QR-factorization with FDK and conjugate gradient (CG) methods show that QR-factorization is able to reconstruct more detailed images for a fixed voxel size.
\end{abstract}

Index Terms-Computed tomography, FDK, medical imaging, Conjugate gradient (CG), RTK toolkit, 3D images reconstruction, QR-factorization algorithm, reconstruction algorithms.

\section{INTRODUCTION}

$\mathrm{T}_{\mathrm{n}}^{\mathrm{H}}$ HE image reconstruction problem in CT can be formulated mathematically as an inverse problem. It is well known that there exists no theoretically exact solution that can stably reconstructs the image from projection data acquired in the commonly used circular trajectory CT [1]. As a result, the

This work was supported in part by the Spanish Government grant TEC2016-79884-C2 and RTC-2016-5186-1

M. J. Rodríguez-Alvarez (e-mail: mjrodri@i3m.upv.es), F. Sánchez, L Moliner, S. Sánchez and J. M. Benlloch are with Instituto de Instrumentación para la Imagen Molecular (I3M). Universitat Politecnica de València (UPV)- majority of one-shot image reconstruction algorithms, such as Feldkamp, Davis and Kress (FDK) [2] and its derivatives, for this configuration are approximate. Another interesting approach is the use of iterative methods, where the reconstruction problem can be described by a discrete linear system [3], which are also approximate methods.

The main disadvantage in iterative methods compared to analytic methods is that the computational cost is higher [3] [5]. Fundamental advances have been made and various methods have been proposed to improve the speed and quality of image reconstruction for medical [6]-[9] and industrial CTs [10]. These methods have shown good results in terms of speed and accuracy of the reconstructed images. Techniques like Simultaneous Algebraic Reconstruction Technique (SART) [11] and Least Square (LSQR) [12] combined with Fast Iterative Shrinkage-Thresholding Algorithms (FISTA) [13], [14] have been proposed to overcome the problem of image reconstruction when few projections are used. Notwithstanding those positive strides, FDK is still accepted as the method of reference in computed tomography [15].

Singular Value Decomposition (SVD) [15]-[17] and QRfactorization [18] have already begun to be investigated as a new arising alternative to the traditional methods. QRfactorization has already been used in medical imaging with promising results [19], [20] but, to date, no satisfactory results have been found for such a large linear systems as required for a 3D image of a CT (in the order $10^{6}$ unknowns).

This paper describes the novel use of QR-factorization of the system matrix of a computed tomography (CT) system, with the motivation to achieve a technique that combines advantages of the traditional method so far used for CT image reconstruction, such as FDK, and by using the system matrix, it allows some of the advantages of iterative methods [3]. Two methods have been used to compare the results obtained with QRfactorization method: FDK, as the traditional method in CT image reconstruction [3] and [15]; and conjugate gradient algorithm (CG) [21], [22], with the implementation of the

Consejo Superior de Investigaciones Científicas (CSIC), Camino de Vera s/n, 46022 Valencia, Spain.

A. Soriano is with the Departament d'Informàtica. Universitat de València. Av de la Universitat s/n. E-46100, Burjassot. Spain. 
reconstruction toolkit (RTK) [23]. CG was chosen because is a commonly used method in CT image reconstruction and it allows us to use the same system matrix than in the case of QRfactorization.

The main advantages of FDK are the computing speed, simplicity and robustness; CG method allows us to use a discrete linear system, which takes into account the scanner geometry, the physical processes involved in the measurements, and it is more suitable for the reconstruction of images with high contrast and precision [4] and [5].

QR-factorization method could be a potential alternative reconstruction method for $\mathrm{CT}$, incorporating the advantages of both methods. The most CPU time-consuming part of the QRfactorization method is calculated and stored only once for a given CT system, and from then on, each image reconstruction only involves a matrix vector product and a backward substitution process. The authors have obtained promising results [24], [25] for the studies of the QR-factorization stability including the errors introduced by the reconstruction process. Additionally, a heuristic has been described in [25] to exploit the particular CT sparse matrix structure allowing a reduction in the number of projections, without compromising image quality.

The reconstructed image obtained with QR-factorization is unique and equivalent to the solution obtained with the least squares method as long as the corresponding system matrix is injective [25]-[27].

Different features have been be studied and compared:

- Noise, which is a key characteristic in image analysis [28]. Two main approaches have been considered to address this issue: 1) standard deviation related analysis: this evaluation will be performed using two figures of merit, namely coefficient of variation (CV) [29] and contrast to noise ratio (CNR) [30]; and 2) spectral analysis, which will be evaluated by means of the noise power spectrum (NPS) [30], [31].

- Sharpness, which will be studied applying a Sobel filter [32] to the reconstructed images.

\section{MAterials AND Methods}

Images of a CT phantom and a mouse were collected on an Albira CT [33] (Fig. 1) and three dimensional (3D) images obtained with QR-factorization have been analysed and compared with those obtained with FDK and CG methods (Fig. 1).

\section{A. Reconstruction methods}

QR-factorization and CG algorithms are model based methods. These model based algorithms consider that image reconstruction in CT can be modelled by a large sparse linear system of equations such as:

$$
A x=b
$$

where $A$ is the system matrix, of dimensions $M \times N, M$ rows and $N$ columns; $b$, of dimensions $1 \times M$, contains the detector element measurements; and $x$, of dimensions $N \times 1$, is the $a$ priori unknown image [25].

Previous to explaining the way the methods were implemented, we are going to give a brief scheme on how the system matrix is constructed.

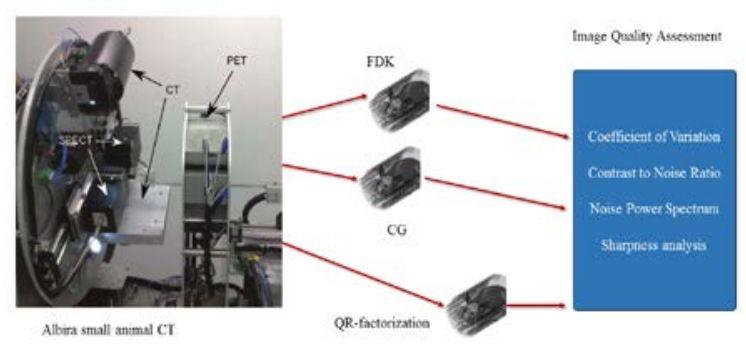

Fig. 1. The CT measurements have been done with the tri-modal Albira PET/SPECT/CT. Three reconstruction methods have been used and compared: FDK, CG and QR-factorization.

\section{1) The system matrix}

The system matrix that describes the scanning process has a major impact on the quality of the reconstructed image [34]. There are two main points to take into account for designing the system matrix:

1. The linear system must be stable.

2. It is desirable that the linear system contains enough information for the image reconstruction.

The first point is related, among others, with how good is the discretization of the CT system. It is necessary to choose the discretization appropriately for obtaining a stable system matrix, which keeps the condition of the matrix down: If $a_{i j}$, denotes the element of the matrix $A$ (i-th row and $j$-th column), then each $a_{i j}$ is defined as $a_{i j}=c(i, j) v_{i j}$, where $v_{i j}$ is the volume intersection between the $i$-th X-ray beam and the $j$-th scanned volume voxel. As the size and relative positions of the pixel detectors and the X-ray source are known for each projection, computing each $v_{i j}$ is a matter of computing the volume intersection between a given cube (voxel) and a pyramid such as its base matches a CT detector element and its apex matches the $\mathrm{x}$-ray source (X-ray beam). The term $c(i, j)$ is referred as the cone beam factor [35], and represents the divergence of the Xray cone beam generated in a point source.

With respect to the second point, by enough information we are referring to $A$ having full rank. We need to define a set of projections that produces at least $N$ linearly independent rows in $A$. If there are sufficient projections in the matrix $A$, there will be enough information to uniquely solve the linear system (1), and that implies [25]-[27]:

$$
d \times P>N
$$

where $d$ is the number of detectors considered in the detector panel, $P$ is the number of projections and $N$ is the number of voxels in the field of view. Notice that $M=d \times P$ is the number of detector element measurements.

\section{2) The $Q R$-factorization algorithm}

As stated above, the QR-factorization method for tomographic image reconstruction is not a broadly-applied approach. For this reason, a brief explanation of the procedure is given below. Typically, for the solution of overdetermined 
systems (like here), the "thin QR" factorization [26] is used, where $R$ is an upper triangular matrix that keeps the dimensions of $A, M \times N$, and $Q$ is an $M \times M$ orthogonal matrix. In this case, the inverse of $Q$ does not exist. Also, $Q Q^{t}$ is not the unity matrix, but $Q^{t} Q$ is (for non-square dimension of $A$ ). The general idea for solving the linear system is to perform the QRfactorization of $A$, exploiting the easy calculation of $Q^{t}$ and apply backward substitution to obtain $x$.

QR-factorization is done via CS-Givens rotations [18]. The numerical properties of this transformation are excellent, that is, the computation of the QR-factorization via Givens rotations is normwise and backward stable. More importantly, if the discretized system (1) is stable, and matrix $A$ has full rank, as is the case, (see point 1 of this section), then the QRfactorization give us the unique solution of the least squares method for this system [25], [36], [37].

Consequently, the linear system (1) is overdetermined, and the solution that minimizes the least square problem is:

$$
x=\arg \min _{y}\|A \cdot y-b\|=\arg \min _{y}\left\|R \cdot y-Q^{t} \cdot b\right\|
$$

and it can be obtained in terms of the QR-factorization [26], [27].

Since the QR-factorization is precomputed and stored, image reconstruction only requires $O\left(M \times N+N^{2}\right)$ operations. $M$ is, at most, the total number of samples (pixel detector elements multiplied by the number of projections measured), and $N$ is the total number of voxels in the reconstructed image. The first factor derives from a matrix vector multiplication, which in the worst case would require $O(M \times N)$ operations or less if the sparsity of the original system matrix is exploited. The second factor derives from a backward substitution process that requires $N^{2}$ operations. The two processes are sequential and therefore their computational costs are added. On the overall cost, $M$ is dominant and $M \times N$ is equivalent to the number of operations required to perform the QR-factorization of the system matrix. However, operations count can be substantially reduced if we take full advantage of the system matrix sparsity.

As far as we now, it is the first time that a study of QRfactorization algorithm for CT is done. This paper is the first study of the viability of the use of QR-factorization as a method for CT image reconstruction, so no filter was applied for QRfactorization. The images are shown as the direct result of the QR-factorization algorithm.

3) The FDK algorithm.

Regarding FDK algorithm, the reconstruction filter function we have used in the FDK implementation is a band-limited ramp filter, using a Gaussian apodization function. The apodization filter required by FDK results in an image blurring of the reconstructed image.

4) The CG algorithm.

When the projection data are complete, as in our case, CG implementation can be used [38]. CG is an unconstrained method that gives us the least square solution when data are complete. Both methods, CG and QR-factorization algorithms, use a system matrix for modelling the CT system. The most remarkable difference is that QR-factorization is a direct method and CG is a classic iterative method. CG was implemented using RTK toolkit [23] and 11 iterations. This number of iterations was chosen because, in our case, it maximized the image contrast to noise ratio (CNR).

\section{B. Data Acquisition}

Experimental measurements have been conducted with the CT of the trimodal PET/SPECT/CT Albira scanner [33]. The CT subsystem has a cone-beam configuration that uses a $50 \mathrm{kV}$ microfocus X-ray source with a focal spot size of $35 \mu \mathrm{m}$ and a CMOS flat-panel detector with an active area of $120 \mathrm{~mm} \times 120$ $\mathrm{mm}$ that consists of a $2400 \times 2400$ pixelated array sensor. The nominal resolution is $8 \mathrm{lp} / \mathrm{mm}$ (line pairs per millimeter) although the measured value is about $11 \mathrm{lp} / \mathrm{mm}$. Fixed distances from the $\mathrm{x}$-ray tube to the isocenter $(290 \mathrm{~mm})$ and $\mathrm{x}$-ray detector $(425 \mathrm{~mm})$ lead to a magnification factor of 1.46. CT transaxial field of view (FOV) is roughly $80 \mathrm{~mm}$ in diameter while the axial FOV is $65 \mathrm{~mm}$. Two groups of images have been analysed: phantom measurements and in vivo measurements. In both cases, 3D images were reconstructed using QRfactorization, FDK and CG methods.

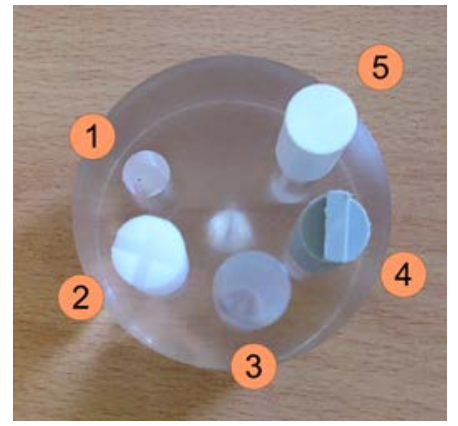

Fig. 2. Phantom with inserts: 1, Air; 2, PFTE; 3, PMMA; 4, PE and 5, POM.

A cylindrical phantom of polymethylmethacrylate (PMMA) $50 \mathrm{~mm}$ height and $55 \mathrm{~mm}$ in diameter was used, see Fig. 2. Five holes of $8 \mathrm{~mm}$ in diameter were axially drilled at $16 \mathrm{~mm}$ off the axis. Three of the holes were filled with $8 \mathrm{~mm}$ in diameter inserts of polyethylene (PE), polyoxymethylene (POM) and Teflon or polytetrafluoroethylene (PTFE), which model adipose tissue, organs tissue and soft bone respectively. A PMMA insert was placed in the third hole, and was used to align the phantom in the scanner. The remaining hole was left empty to model an air region inside the body. A mouse image was also recorded following the procedure described in [25] and [33]. In which follows, we have considered pixel detector size of 0.2 $\mathrm{mm} \times 0.2 \mathrm{~mm}$. Although the actual detector has $50 \mu \mathrm{m} \times 50 \mu \mathrm{m}$ pixelated array, a $4 \times 4$ binning is selected in order to reduce statistical noise.

\section{Image Quality Indicators}

The 3D CT images of the phantom were reconstructed using QR-factorization, FDK and CG methods. The first step for the numerical analysis of the reconstructed images is the definition of meaningful volume of interest (VoI). All VoIs were cylindrical along the axial axis with a height of approximately $38 \mathrm{~mm}$ (in order to maximize the number of considered slices in all image configurations). Moreover, all VoIs have a diameter of $4 \mathrm{~mm}$ (inside each insert which is $8 \mathrm{~mm}$ in 
diameter), so that the $2 \mathrm{~mm}$ outer side of each insert is excluded in order to avoid considering voxels that are part of transition between materials. In this case, the selected VoIs are representative of the different body regions:

- $\quad$ Air, centered in its own insert, will be a measure of high contrast low valued region. Also, this VoI will demonstrate the ability for each algorithm to reproduce empty regions inside the body.

- $\quad$ Bone, centered in the Teflon insert, will be a measure of high contrast high valued region.

- $\quad$ Adipose tissue, centered in the PE insert, will be a measure of low contrast low valued region.

- $\quad$ Organs, centered in the POM insert, will be a measure of low contrast high valued region.

- $\quad$ Soft tissue, PMMA, will be a measure of CT images background that will be used to compute contrast for the different VoI regions.

Two figures of merit have been used to measure the quality of the phantom reconstructed images:

- Coefficient of variation (CV) [9] quantifies the variability among voxel values in a VoI. CV is divided by $\mu_{\mathrm{v}}$ in order to obtain a dimensionless parameter:

$$
C V=\frac{\sigma_{v}}{\mu_{v}} 100
$$

where $\sigma_{\mathrm{v}}$ is the standard deviation of the VoI voxels and $\mu_{\mathrm{v}}$ represents the CT (Hounsfield) number.

- Contrast to noise ratio (CNR) [29] is a measure of the relation between contrast (difference between means) and noise (standard deviation) of a VoI and the PMMA background:

$$
C N R=\frac{2\left|\mu_{v}-\mu_{b}\right|}{\sigma_{v}+\sigma_{b}} 100
$$

where $\sigma_{\mathrm{b}}$ and $\mu_{\mathrm{b}}$ represent the standard deviation and the CT number of the background region, respectively.

CNR and CV parameters complement each other. On one hand, the CNR gives a value of how much a given difference can be seen through the noise that is present. In other words, this parameter answers the following question: will the current noise level let see image features with a certain contrast? The result is a percentage representing the visibility of the considered VoI.

On the other hand, the CV gives the voxel variability independent of its mean value, in order to get the noise values comparable among different VoIs. As our VoIs are defined over homogeneous regions, this parameter accounts directly for the noise present in the reconstructed image. Notice that this value will depend not only on the algorithm used for the image reconstruction, but also on the CT measurement (i.e. the quality of input data). However, differences in this parameter using the same CT data will account for the differences of the algorithms considered when dealing with noise. The combination of these two figures of merit illustrates the amount of noise present and its influence in the interpretation on the reconstructed image.

We have implemented also a multidimensional analysis of the noise power spectrum (NPS), following the guidelines described in [25], [39] and [40]. Volumetric realizations have been extracted from 3D reconstructed images of an empty FOV (air). This results in uniform images that are easily detrended to zero mean images.

The 3D NPS images have been obtained and averaged to avoid statistical fluctuations and, finally, the resulting 3D NPS image has been analysed. Reconstructed images have been converted to CT number, in order to obtain the NPS in the appropriate units.

In order to quantify the blurring of the reconstructed images, several metrics have been proposed in the literature. For example, variance [41] or kurtosis [42], [43] based metrics, among many others [44], have been proposed. However, these metrics are influenced by the amount of noise in the reconstructed image and in our case they are very sensitive to changes in voxel size. One of the effects of blur is the spread of the edges in an image. As it will be seen later, (Results and Discussion section), QR-factorization, FDK and CG show quite different edge spreading in their reconstructed images. For that reason, the analysis of the spread of the edges [45] has been adopted and adapted to our particular case, in order to quantify the image blurring of the reconstruction algorithms. To do that, we have used the Sobel operator [32] which provides a gradient estimate at a slice (2D) image point obtained in a $3 \times 3$ pixels neighbourhood of that point. This operator has been widely used in image processing and nowadays is available in almost every image software.

\section{RESUltS AND DisCUSSION}

\section{A. Phantom results}

Fig. 3 (a)-(h) show the results obtained for the axial central slice of the 3D reconstructed image of the phantom using QRfactorization, FDK and CG for different voxel sizes. Cubic voxels of different edge lengths were used in the image reconstructions. For simplicity it will be used the length of the edge for voxel size labelling. Fig. 3 (i) shows a scheme of the axial, sagittal and coronal slices. Fig. 3 (j)-(k) shows the sagittal and coronal slice of the QR $0.8 \mathrm{~mm}$ voxel size image.

The differences among the used methods of reconstruction in the case of sagittal images or coronal images are similar to those observed in the case of axial images. QR results obtained for $0.8 \mathrm{~mm}$ voxel size are comparable with those obtained with 0.2 $\mathrm{mm}$ voxel size for FDK. Regarding the blur, the main feature of the transition between materials is how quickly it is made. Ideally, transitions should be quickly and therefore, abrupt. However, smooth transitions can be observed, for example, in the case of Teflon insert reconstructed with FDK or air insert reconstructed with $\mathrm{CG}$.

Fig. 4(a) shows the 3D rendering of the 3D reconstruction of the phantom with the QR-factorization algorithm with $0.8 \mathrm{~mm}$ of voxel size. Differences among the 3D renderings with the three reconstruction algorithms only are noticeable at the screw thread. Fig. 4 (b)-(d) show a zoom of the central fastening screw zone (see Fig. 2) with $0.8 \mathrm{~mm}$ of voxel sixe for QRfactorization, FDK and CG reconstruction algorithms 
respectively. It can be observed that for $0.8 \mathrm{~mm}$ of voxel size, only QR-factorization and CG algorithms are able to properly reproduce the screw thread. Fig. 4 (e) shows that FDK is also able to reconstruct the screw zone with $0.2 \mathrm{~mm}$ of voxel size.

In terms of visual (subjective) quality, the QR reconstruction with $1.2 \mathrm{~mm}$ voxels size can be placed in between the 0.2 and $0.8 \mathrm{~mm}$ voxel size reconstructions of FDK and CG. Likewise, the QR reconstruction with $0.8 \mathrm{~mm}$ voxel size produces even better visual quality image than the $0.2 \mathrm{~mm}$ voxel size reconstructions with FDK and CG.

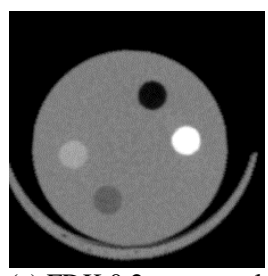

(c) FDK $0.2 \mathrm{~mm}$ voxel size

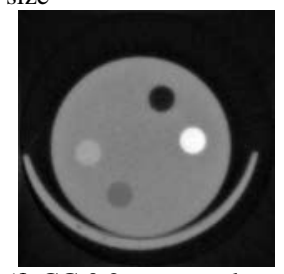

(f) CG $0.2 \mathrm{~mm}$ voxel

size
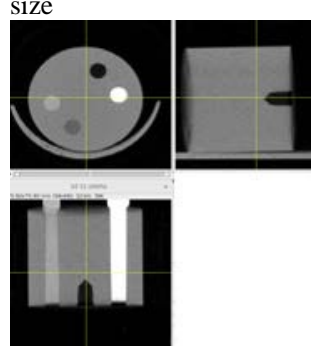

(i) Scheme of the axial, coronal and sagittal views.

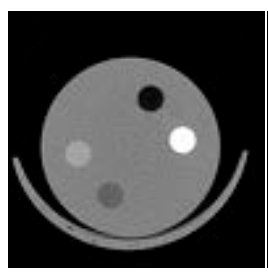

(a) QR $0.8 \mathrm{~mm}$ voxel size

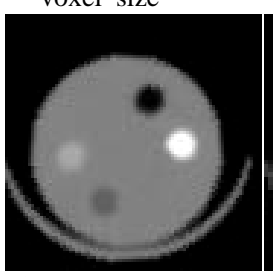

(d) FDK $0.8 \mathrm{~mm}$ voxel size

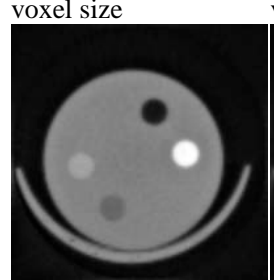

(g) CG $0.8 \mathrm{~mm}$ voxel size

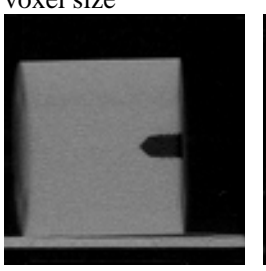

(j) Sagittal slice of the QR $0.8 \mathrm{~mm}$ voxel size image

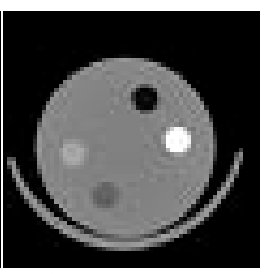

(b) QR $1.2 \mathrm{~mm}$ voxel size

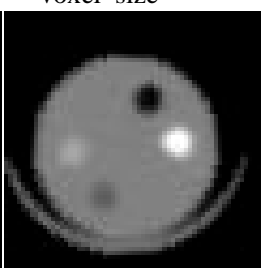

(e) FDK $1.2 \mathrm{~mm}$ voxel size

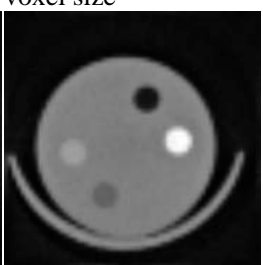

(h) CG $1.2 \mathrm{~mm}$ voxel size

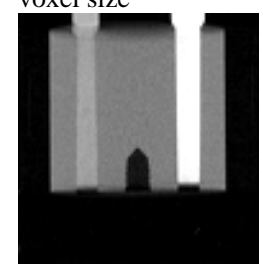

(k) Coronal slice of the QR $0.8 \mathrm{~mm}$ voxel size image
Fig. 3 (a)-(h) Central axial slice of the 3D reconstructed image of the phantom. Differences in the edge definition can be perceived even with a naked eye. (i), (j) and (k) are the axial, sagittal and coronal views of the QR $0.8 \mathrm{~mm}$ voxel size image. Only in the case of QR reconstruction, sagittal and coronal views have been included. The phantom inserts orientationlocation is the same already shown in Fig. 2

This is significant in the sense that voxel size has an important role in the degradation of the image quality. In this case, a large voxel size $(1.2 \mathrm{~mm})$ refers to the relation between the voxel edge size and each insert diameter $(8 \mathrm{~mm})$ which leaves 6 voxels in diameter for each insert. With so few voxels, the reconstruction algorithms should perform a finer estimation of the attenuation coefficients, in order to correctly translate curved edges (between materials) and avoid blurring.

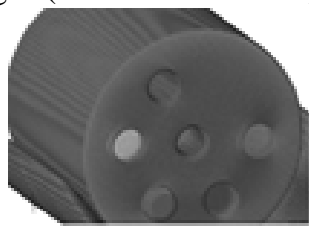

(a) 3D QR $0.8 \mathrm{~mm}$ voxel size.

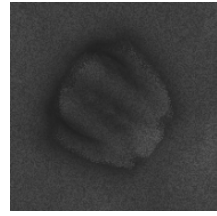

(c) Zoom of the screw thread zone FDK $(0.8$ mm voxel size).

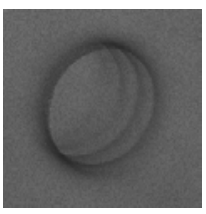

(d) Zoom of the screw thread zone CG $(0.8$ mm voxel size)

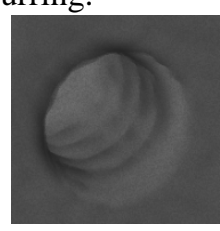

(b) Zoom of the screw thread zone QR $(0.8$ mm voxel size).

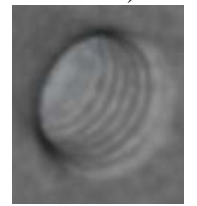

(e) Zoom of the screw thread zone FDK $(0.2$ $\mathrm{mm}$ voxel size).
Fig. 4. (a) 3D phantom image reconstructed with QR algorithm. Differences among the three reconstruction algorithms only are easily noticeable in the screw thread with a zoom. (b)-(e) Zoom of the screw thread zone of the phantom.

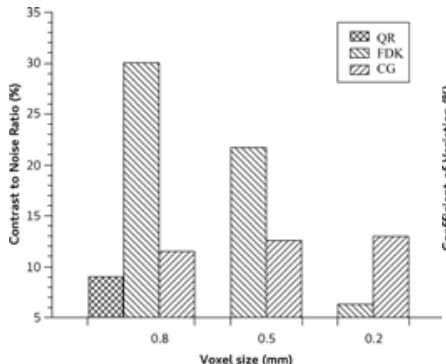

(a)

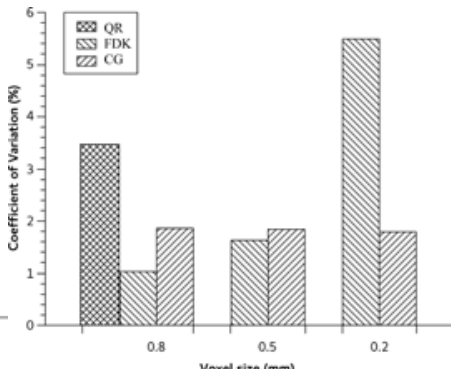

(b)
Fig. 5. CNR (a) and CV (b) of the PE VoI for each algorithm and voxel size. CV is presented alongside CNR in order to highlight noise contribution to CNR worsening.

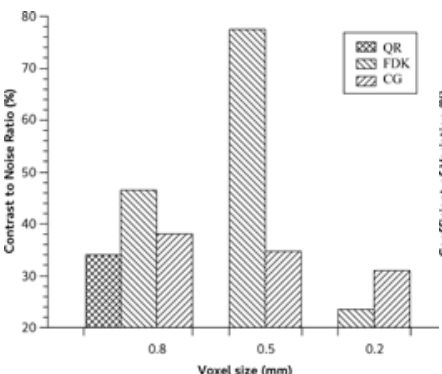

(a)

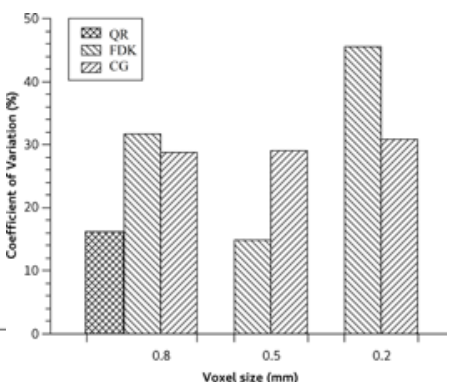

(b)
Fig. 6. CNR (a) and CV (b) of the air VoI for each algorithm and voxel size. $\mathrm{CV}$ is presented alongside CNR in order to highlight noise contribution to CNR worsening

This effect is mostly appreciated in the FDK algorithm with $1.2 \mathrm{~mm}$ voxels and it is not that evident in the QR-factorization and CG algorithms. The better performing of QR and CG is mainly due to the fact that they are model based methods. 


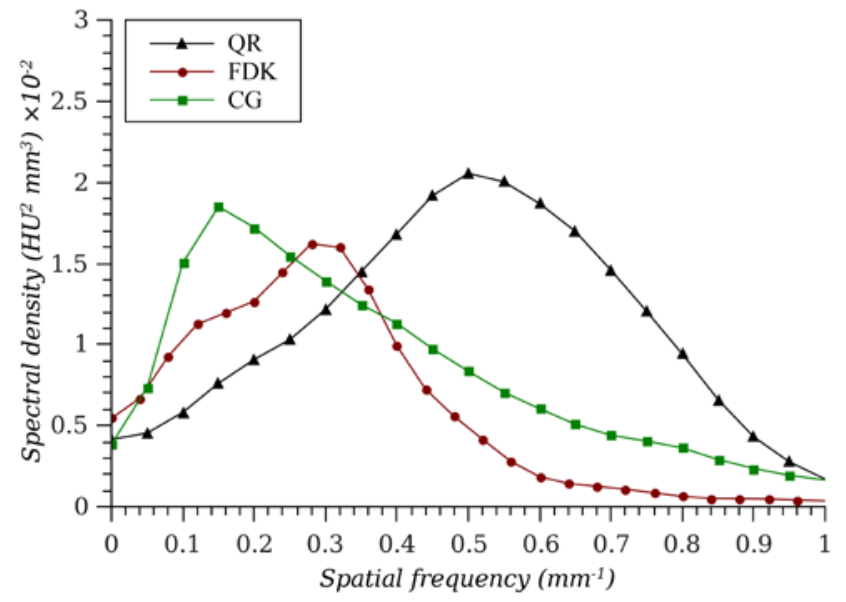

Fig. 7. NPS comparison among images reconstructed with QR, FDK and CG with $1.2 \mathrm{~mm}$ voxel size.

In Fig. 5 results for CNR and CV measured in PE, low contrast VoIs, are presented. The degradation in CNR results is due to the increase in CV. The comparison between all the three algorithms shows that QR has the poorest CNR results. CNR and CV results obtained with QR (0.8 $\mathrm{mm}$ voxel size) are only comparable to those obtained with FDK or CG using $0.2 \mathrm{~mm}$ voxel size.

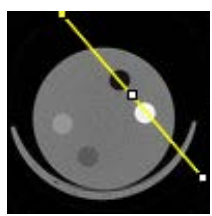

(a)

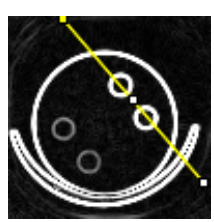

(b)

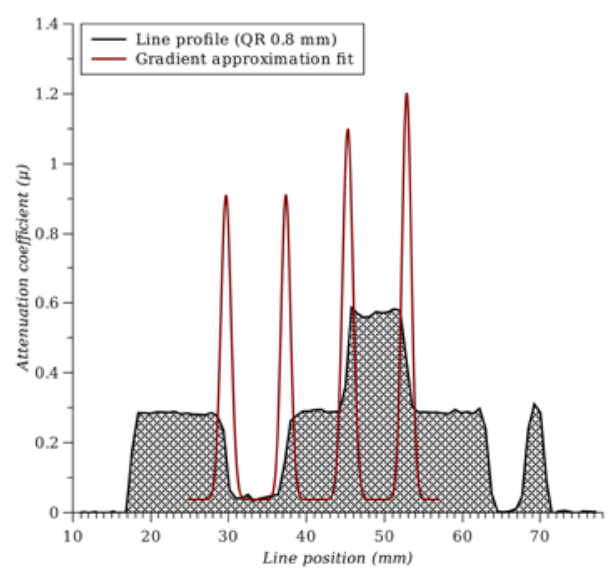

(c)
Fig. 8. Characterization of transitions among different phantom inserts materials. In this example, image has been reconstructed with QRfactorization using $0.8 \mathrm{~mm}$ voxel size. A line profile through the high contrast inserts at central slice (a) is selected, and shown as a filled curve in (c). In the first valley (around $35 \mathrm{~mm}$ ) is located the air insert and in the first peak (around $50 \mathrm{~mm}$ ) is located the Teflon insert. A Sobel operator is applied to the central slice to obtain a gradient estimate image (b) and the previous line profile is reevaluated to yield gradient estimates. Only the line segment between 25 and $57 \mathrm{~mm}$ (where the transitions involving inserts are located) is considered. Four Gaussian distributions are fitted to this line segment and shown in (c). The FWHM of these Gaussian distributions are associated to the transition widths.

The air VoI presents a different case (see Fig. 6), mainly due to the fact that there is a great influence of noise in the air VoI due to its low attenuation coefficient. Moreover, for the rest of inserts considered in the phantom (PTFE, PMMA, PE, and $\mathrm{POM}$ ), the CV obtained with FDK increases as the voxel size decreases. However, for the air insert, we notice that the minimum CV for the FDK is obtained with $0.5 \mathrm{~mm}$ voxels (see Fig. 6). We have not found so far any clear reason capable to explain this behaviour.

TABLE I

AVERAGED FWHM OF THE FITTED GAUSSIANS DISTRIBUTION TO THE SOBEL GRADIENT ESTIMATES.

\begin{tabular}{|c|c|c|c|}
\hline Material & Method & $\begin{array}{l}\text { Voxel }^{\mathrm{a}} \\
\text { Size } \\
(\mathrm{mm})\end{array}$ & $\begin{array}{c}\text { FWHM }^{\mathrm{b}} \\
(\mathrm{mm})\end{array}$ \\
\hline \multirow[t]{7}{*}{ Air } & $\overline{Q R}$ & 0.8 & 1.5 \\
\hline & CG & 0.8 & 1.9 \\
\hline & & 0.5 & 1.4 \\
\hline & & 0.2 & 1.0 \\
\hline & FDK & 0.8 & 2.3 \\
\hline & & 0.5 & 1.6 \\
\hline & & 0.2 & 0.5 \\
\hline \multirow[t]{7}{*}{ Teflon } & QR & 0.8 & 1.5 \\
\hline & CG & 0.8 & 1.8 \\
\hline & & 0.5 & 1.4 \\
\hline & & 0.2 & 0.9 \\
\hline & FDK & 0.8 & 2.6 \\
\hline & & 0.5 & 1.6 \\
\hline & & 0.2 & 0.6 \\
\hline \multirow[t]{7}{*}{ PE } & QR & 0.8 & 1.3 \\
\hline & CG & 0.8 & 1.9 \\
\hline & & 0.5 & 1.5 \\
\hline & & 0.2 & 0.6 \\
\hline & FDK & 0.8 & 2.5 \\
\hline & & 0.5 & 1.4 \\
\hline & & 0.2 & 0.5 \\
\hline \multirow[t]{7}{*}{ POM } & QR & 0.8 & 1.4 \\
\hline & CG & 0.8 & 2.2 \\
\hline & & 0.5 & 1.5 \\
\hline & & 0.2 & 0.6 \\
\hline & FDK & 0.8 & 2.5 \\
\hline & & 0.5 & 1.7 \\
\hline & & 0.2 & 0.5 \\
\hline
\end{tabular}

${ }^{\mathrm{a} C u b i c}$ voxels of different edge lengths have been used in the image reconstructions. For simplicity it has been used the length of the edge for voxel size labelling.

${ }^{b}$ The averaged FWHM for each material is the mean value of its two FWHM (corresponding to the transition inside and outside the insert for a selected line profile in the central slice, see Fig. 9 and 10).

Fig. 7 shows the result of the NPS for QR, FDK and CG. FDK exhibits the expected behaviour. QR produces lower values than FDK and CG at low frequencies while for higher spatial frequencies it produces higher values than FDK and CG. This difference is associated with the preservation of high spatial frequencies by the QR-factorization algorithm and therefore although fine details in the image are preserved, producing higher sharpness (see Fig. 3 (a)) than FDK and CG, it produces fine noise too, as can be seen in Fig. 7.

In Fig. 8 the sharpness (in terms of the spread of the phantom edges concerning transitions among different phantom inserts) is characterized and quantified for the QR-factorization algorithm using $0.8 \mathrm{~mm}$ voxel size. The Sobel filter was applied to the original image (Fig. 8 (a)), obtaining the gradient estimate image (Fig. 8 (b)) in which the transitions between different insert materials can be easily characterized. Fig. 8 (c) shows the line profile: the four central peaks (transitions into and out of the inserts) are selected and then four Gaussian distributions are 
fitted to them (as seen in Fig. 8 (c)). The Full Width at Half Maximum (FWHM) of these distributions represents the distance needed for the reconstruction algorithm to perform the transition between different materials. The two FWHMs of the two Gaussian distributions associated to the transitions involving an insert are averaged, in order to obtain a single value for each material.

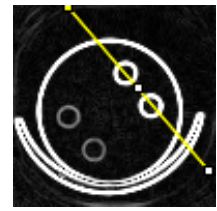

(a)

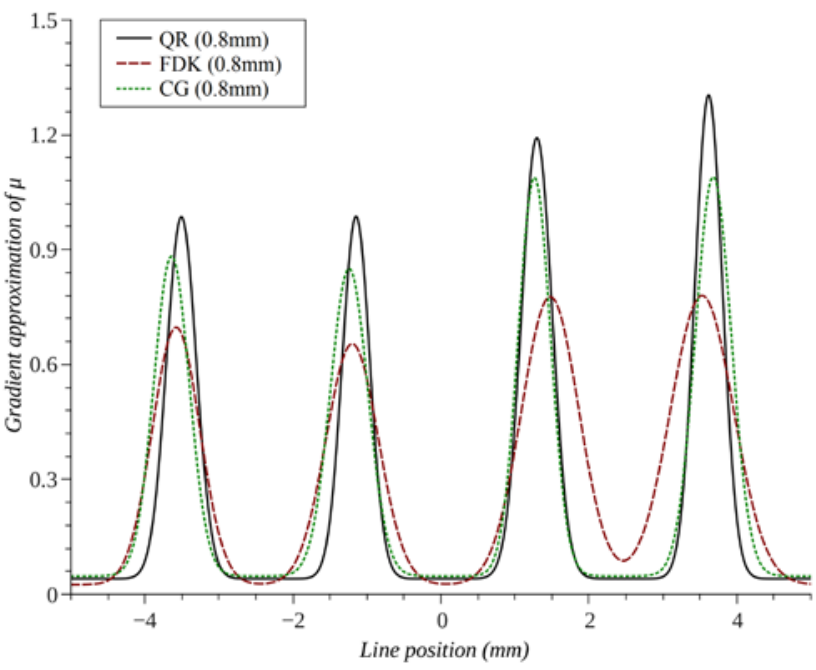

(d)

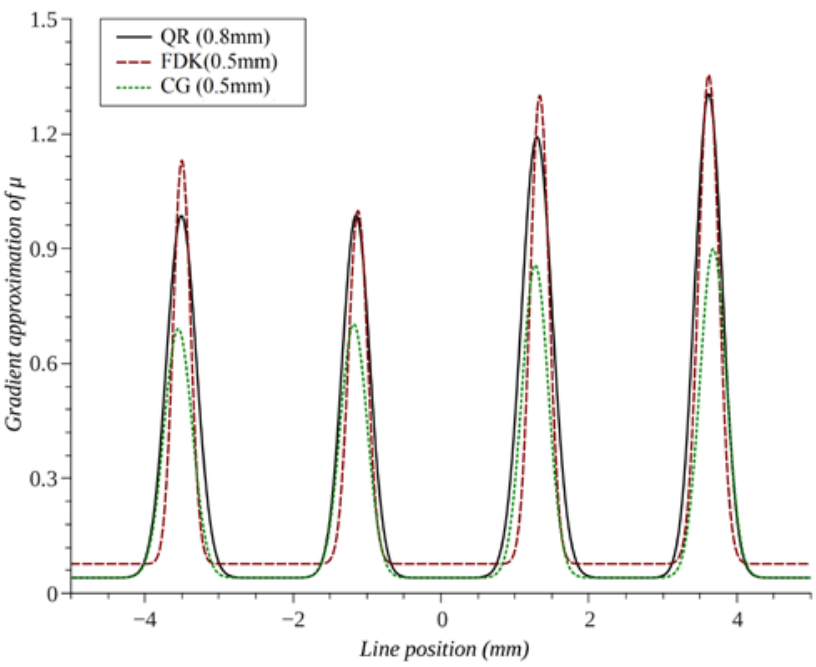

(e)

Fig. 9. Gradient estimate fitting through a line profile for high contrast inserts (air and Teflon) from images reconstructed with QR (a), FDK (b) and CG (c) algorithms. In d) the image voxel size is the same $(0.8 \mathrm{~mm})$, while in e) the image voxel size for QR $(0.8 \mathrm{~mm})$ is different from that used for FDK and CG (0.5 mm).

The same procedure was applied to images reconstructed with FDK and CG algorithms. Notice that the procedure is defined for a given line profile. However we have verified that different line profiles produce similar FWHM values. The
Sobel gradient estimates fitted to Gaussian distributions, both to the high contrast gradients (air and Teflon) and to the low contrast gradients (PE and POM) are shown in Fig. 9 and 10, respectively.

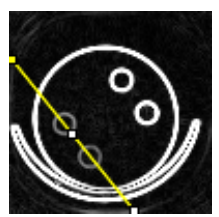

(a)

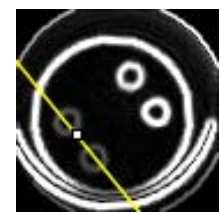

(b)

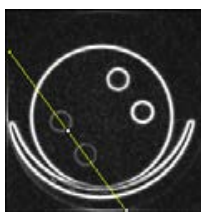

(c)

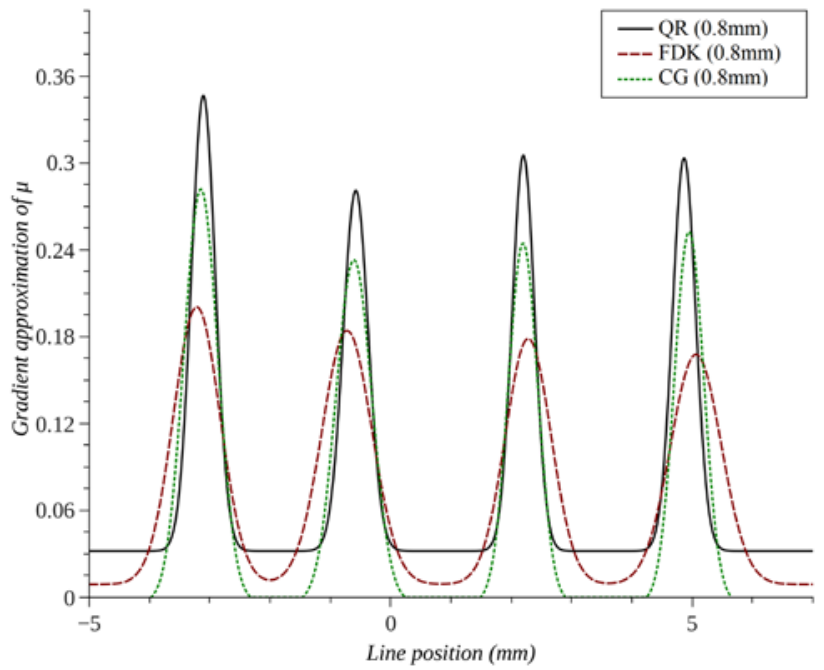

(d)

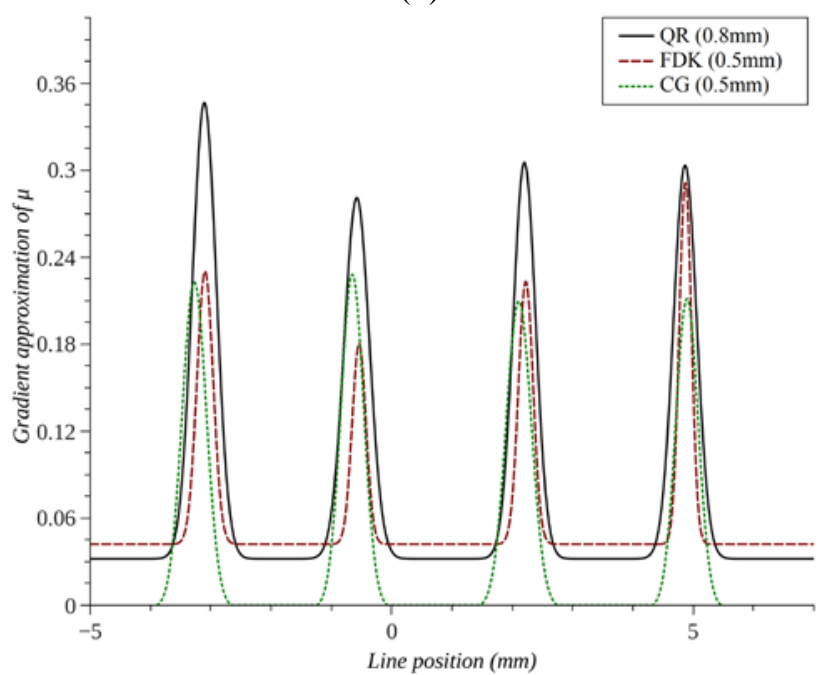

(e)

Fig. 10. Gradient estimate fitting through a line profile for low contrast inserts (PE and POM) from images reconstructed with QR (a), FDK (b) and CG (c) algorithms. In d) the image voxel size is the same $(0.8 \mathrm{~mm})$, while in e) the image voxel size for QR $(0.8 \mathrm{~mm})$ is different from that used for FDK and CG (0.5 mm).

In our case, the most important parameter of the gradient peaks is their width (FWHM). Thin peaks represent short transitions and therefore, sharp edges. In Fig. 9 and 10 the different peaks represent, from left to right, transitions inside and outside the first insert and inside and outside the second insert (air and Teflon in the high contrast case, Fig. 9, and POM 
and PE in the low contrast case, Fig. 10). In all cases, for the same voxel size, QR-factorization algorithm produces thinner gradients, i.e. sharper transitions. Gradients produced by QR using $0.8 \mathrm{~mm}$ voxel size are equivalent to those produced by FDK or CG using $0.5 \mathrm{~mm}$ voxel size. It should be noticed that despite of the fact that in some cases FDK and CG produce thinner gradients than $\mathrm{QR}$, this implies a considerable increase in the image size (from $0.8 \mathrm{~mm}$ to $0.5 \mathrm{~mm}$ voxel size). In other words, with fewer voxels, QR is able to produce transitions as narrow as FDK or CG with almost half sized voxels.

This is confirmed in the summary of the Sobel analysis we show in Table 1, where the FWHM of the fitted Gaussians distribution to the Sobel gradient estimates, for the different algorithms, voxel sizes, and phantom insert materials considered in this work are shown.

The FWHM we show in Table 1 should be considered as an estimator that remains almost invariable to changes in the noise level. From Table 1 we can conclude that QR reproduces image edges as sharp as FDK or CG but using almost half the voxel size. Notice that image resolution is influenced by the sharpness of the edges, in the sense that spatially close regions belonging to two different anatomical features could be indistinguishable if their transitions are smooth enough.

\section{B. In Vivo Results}

In order to verify the previously results in a more realistic situation, in vivo measurements of a mouse have been reconstructed with all three methods (QR, FDK and CG), using $0.8,0.5$ and $0.2 \mathrm{~mm}$ voxel sizes. Comparisons were carried out selecting a line profile through a fine feature region in the knee of the mouse (see Fig. 11).

The same slice in the axial direction has been selected in all images, yielding a cross section in the mouse femur, tibia and fibula. Considering the size of the detectors $(0.2 \mathrm{~mm})$ used for these measurements, the soft bone tissue of the mouse femur ( 1 $\mathrm{mm}$ ) should be clearly visible, surrounded by the femur hard bone tissue (approximately $1 \mathrm{~mm}$ to the left and to the right) and hard bone tissue for the tibia and fibula $(0.7 \mathrm{~mm})$. The femur has been considered as a coarse feature and the fibula has been considered as a fine feature. A line profile through the femur and fibula is enough to observe the presence of these coarse and fine features. This line profile should present peaks in hard bone regions and valleys in soft bone regions (or between bone pieces).

Considering the mouse image slice and line profile analysed in this section, three different resolution levels could be defined. The first one, in which the femur is clearly seen (3 mm total width), although its interior, soft bone tissue, is not observable. This is partly due to the spread of the edges of the hard bone peaks. The second one, in which the femur and its interior, soft bone tissue, is observable, but the fibula is not. This is partly due to the impossibility of the production of thin peaks with smooth transitions. And the third one, in which all features are observable, namely, femur soft and hard bone and fibula hard bone tissues.

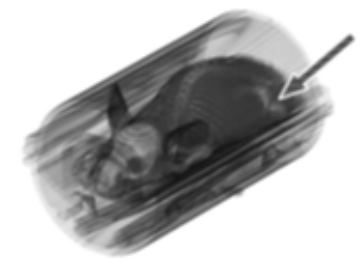

(a)
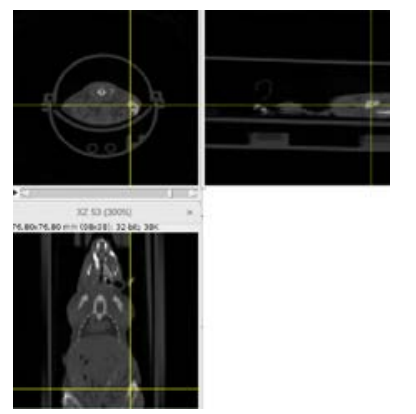

(c)

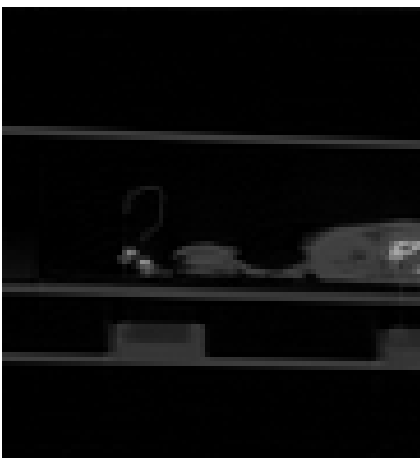

(e)

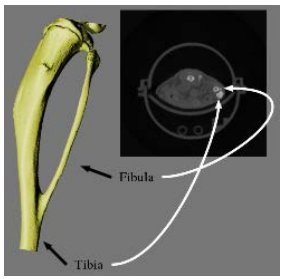

(b)

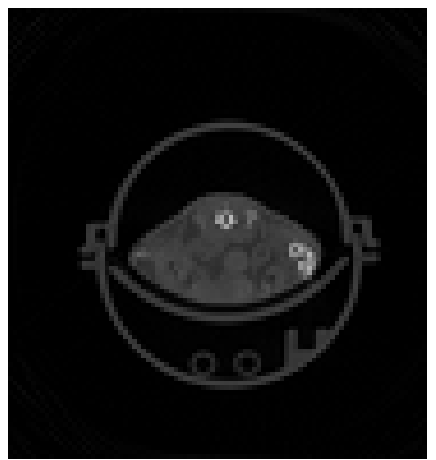

(d)

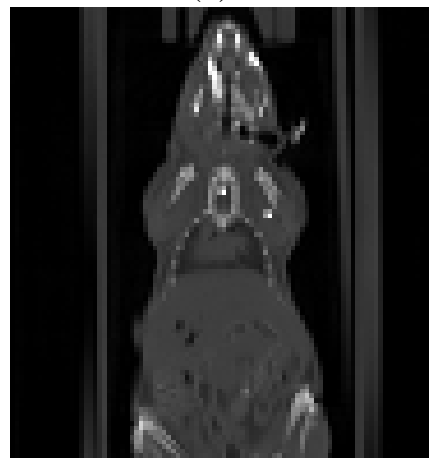

(f)
Fig. 11. 3D reconstruction of a mouse using QR-factorization method and $0.8 \mathrm{~mm}$ voxel size. The entire mouse is shown in (a) and the mouse left knee is pointed by an arrow. The mouse is on a stretcher that provides it with anesthesia. (b) axial view of the mouse showing the fibula and tibia bones marked by the arrow in (a). (c) Shows a scheme of the axial, coronal and sagittal views. (d), (e) and (f) are the axial, coronal and sagittal images respectively.

The results of this test are summarized in Fig. 12. As previous results indicated, QR with $0.8 \mathrm{~mm}$ voxel size and CG with 0.5 $\mathrm{mm}$ voxel size have comparable sharpness and almost the same features can be observed, see Fig. 12 (a) and (b). As we already stated in the phantom measurements section, QR-factorization method can produce images as sharp and rich in features as CG, but CG needs almost twice voxel size. FDK algorithm with 0.5 mm voxel size, see Fig. 12 (c), produces the worst result. Visually, FDK slice presents a lower edge definition than QR and CG and, therefore, less features can be observed, unless 0.2 $\mathrm{mm}$ voxel size is used, Fig. 12 (d).

In Fig. 12 (e)-(f) attenuation profiles (in Hounsfield units) for FDK and CG are compared with that obtained with QR method. The hard bone peaks produced by QR-factorization algorithm are not exactly where they should, due to the undersampling produced for the use of large voxel sizes. CG and FDK methods show a peak in the fibula region for the smaller voxel size $(0.2$ $\mathrm{mm}$ ) and all the three algorithms are capable to reproduce the 
femur soft bone tissue. Using almost twice the voxel size, QRfactorization algorithm reconstructs even a sharper image than FDK, Fig. 12 (e). The main numerical difference arises in the transition involving the femur soft bone tissue. While QR is able to reproduce it, the FDK reconstruction with voxels sizes ranging from 0.8 to $0.5 \mathrm{~mm}$, is not. It is necessary to use FDK with $0.2 \mathrm{~mm}$ voxel size to properly reproduce the femur soft bone tissue, but as already mentioned this implies a considerable increase in the image size.

\section{Execution time.}

The image reconstruction times have been measured in a computer with an Intel Core i7 with 8 threads at $4 \mathrm{GHz}$ clock frequency. RTK toolkit is parallelized into 8 threads; the CG reconstruction with 11 iterations needed $94.6 \mathrm{~s}$ for $1.2 \mathrm{~mm}$ voxel size and $146.9 \mathrm{~s}$ for $0.8 \mathrm{~mm}$ voxel size. With respect to QR-factorization algorithm, it is necessary to point out that the reconstruction time accounts for $Q^{t} \cdot b$ matrix vector multiplication plus the backward substitution process; the time for the backward substitution process with a parallelization of 7 threads is $12.0 \mathrm{~s}$ for $1.2 \mathrm{~mm}$ of voxel size and $96.3 \mathrm{~s}$ for $0.8 \mathrm{~mm}$ of voxel size, and computation time for the matrix vector multiplication $Q^{t} \cdot b$ (without parallelization) is $80.0 \mathrm{~s}$ for $1.2 \mathrm{~mm}$ voxel size and $6050 \mathrm{~s}$ for $0.8 \mathrm{~mm}$ voxel size. The reconstruction time, in the case of CG, increases a factor of 1.5 when the voxel size decreases $1 / 3$, while in the case of QR-factorization this factor is 75 . This is due to the increase in the number of fill-in of the matrix that depends on the structure of the matrix, i.e. the increase is not linear. Finally, as expected, FDK reconstruction time with 8 threads is $0.25 \mathrm{~s}$ for $1.2 \mathrm{~mm}$ voxel size and $0.65 \mathrm{~s}$ for $0.8 \mathrm{~mm}$ voxel size.

\section{CONCLUSIONS}

During decades, FDK has been the standard method used for tomographic imaging reconstruction. However, as the image reconstruction problem can be modelled by a large linear system of equations, and taking into account the current advances in computer science, QR-factorization of the system matrix could be considered as a serious competitive method in reconstruction of tomographic images. Main advantage of the proposed QR-factorization method is that the core of the algorithm, from the computational cost point of view, is precalculated and stored only once for a given system, and from then on, each image reconstruction only involves a backward substitution process. We have developed the QR-factorization method for CT system and we have compared the images obtained with FDK and CG using real data from a preclinical CT system. Comparison between QR, FDK, and CG for images of a phantom with different material inserts show that QRfactorization provides more information for a fixed voxel size, providing more detailed images and introducing less blurring in the reconstructed image, see Fig. 4 (e)-(h). NPS analysis shows that QR produces higher values than FDK and CG at higher spatial frequencies and therefore although fine details in the image are preserved, producing higher sharpness (see Fig. 3 (a)) than FDK and CG, it produces fine noise too (Fig. 7).

Reconstructed images sharpness has been quantified using the Sobel operator, which provides a gradient estimate for a given 2D slice. The Sobel gradient estimate shows that QR reproduces image edges as sharp as FDK and CG using almost half the voxel size. It should be pointed out that QR method not only is able to obtain higher gradient magnitude than FDK and CG, which is related to fast change between image values in Hounsfield units, but also the peak width of the gradients (FWHM) for QR reconstructed images are thinner than those of FDK and CG. The peak widths are directly related to the ability of the algorithm when dealing with border zones between different materials, i.e. regions with different $\mathrm{X}$-ray absorption. In vivo measurements of a mouse confirm the results we have obtained for the phantom, i.e. for a fixed voxel size QR outperforms the other two algorithms considered in this work (CG and FDK). In this sense, we could conclude that the QRfactorization algorithm is more efficient in terms of image features (image information). Although QR-factorization algorithm has not yet been depurated in terms of execution time, run time of QR-factorization algorithm is comparable to that needed for CG for $1.2 \mathrm{~mm}$ of voxel size, assuming 11 iterations for CG. Future work will include QR time execution optimization by parallelization of the different processes of the algorithm and new strategies for reduction of the amount of fillin produced during the reduction of the system matrix to the triangular form.

The use of the QR-factorization algorithm for tomographic image reconstruction is just starting. The obtained results encourage us to further development of this technique to overcome the challenges we currently face. This will lead to the development of a reconstruction algorithm for tomographic image reconstruction with the advantages of FDK, like the speed and robustness; and the advantages of iterative algorithms, by using the system matrix, where fully physical information of the system can be included. 


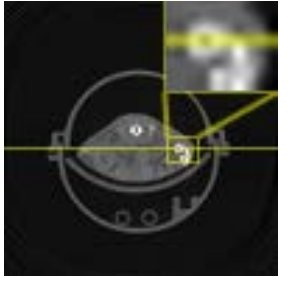

(a)

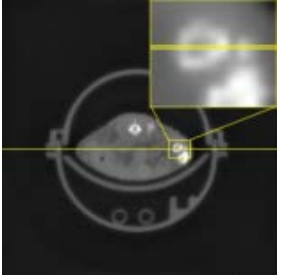

(b)

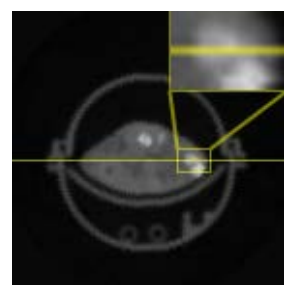

(c)

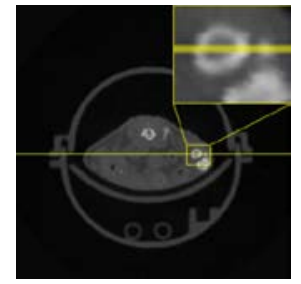

(d)

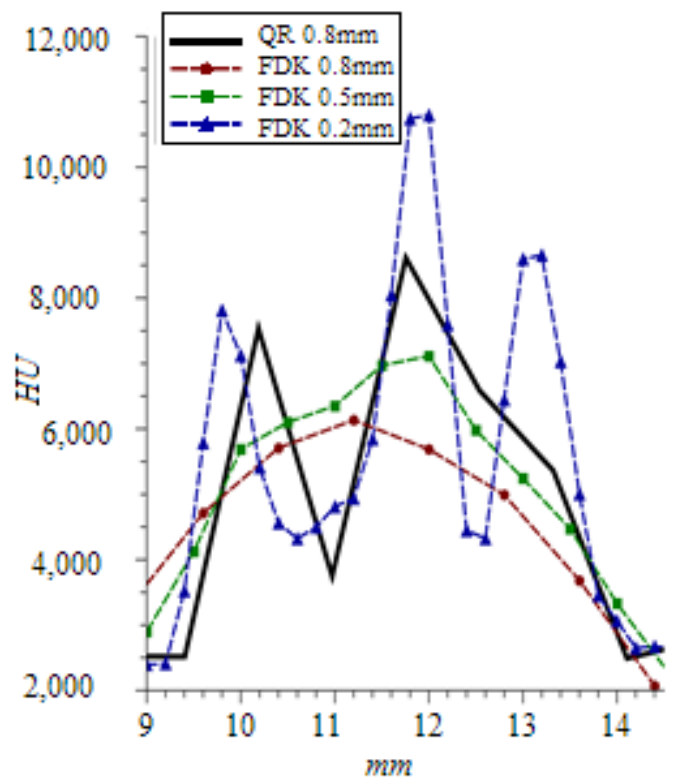

(e)

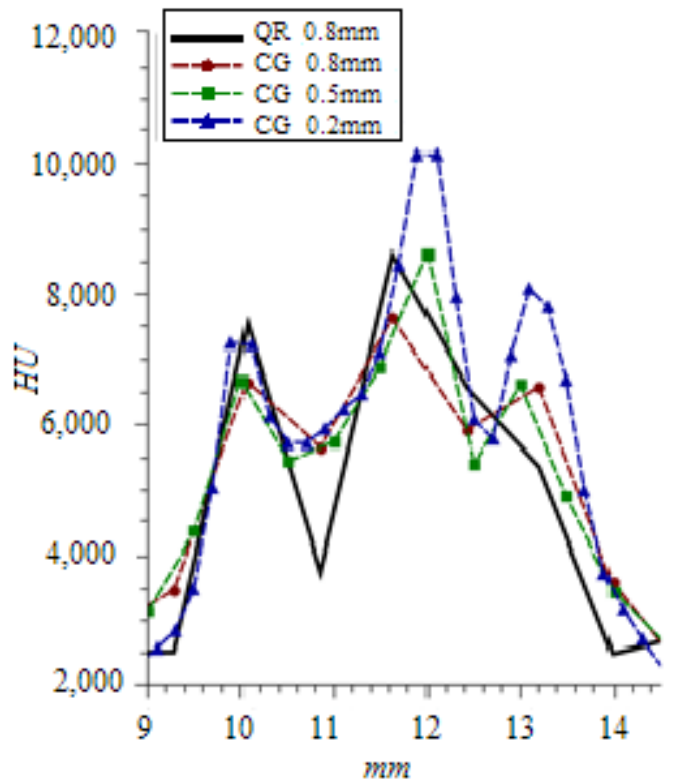

(f)

Fig. 12. Line profiles through mouse femur and fibula axial slice. The slice has been selected from a 3D image reconstruction using: (a) QR with $0.8 \mathrm{~mm}$ voxel size, (b) CG with $0.5 \mathrm{~mm}$ voxel size, (c) FDK with $0.5 \mathrm{~mm}$ voxel size, and (d) FDK with $0.2 \mathrm{~mm}$ voxel size. Attenuation profiles (in Hounsfield units) for FDK (e) and CG (f) using different voxel sizes are compared with that obtained with QR for $0.8 \mathrm{~mm}$ voxel size. Profile plots begin right before entering the femur and end right after leaving the fibula.

\section{REFERENCES}

[1] H.K. Tuy, "An Inversion Formula for Cone-Beam Reconstruction,” SIAM J. Appl. Math. 43 (1983) 546-552. doi:10.1137/0143035.S.

[2] L. A. Feldkamp, L. C. Davis, and J. W. Kress, "Practical cone-beam algorithm,” J. Opt. Soc. Am. A, vol. 1, no. 6, p. 612, Jun. 1984.

[3] L. L. Geyer, U. J. Schoepf, F. G. Meinel, J. W. Nance, G. Bastarrika, J. A. Leipsic, N. S. Paul, M. Rengo, A. Laghi, and C. N. De Cecco, "State of the Art: Iterative CT Reconstruction Techniques,” Radiology, vol. 276, no. 2, pp. 339-357, Aug. 2015.

[4] L. A. Flores, V. Vidal, P. Mayo, F. Rodenas, and G. Verdú, “CT Image Reconstruction Based on GPUs,” Procedia Comput. Sci., vol. 18, pp. 1412-1420, 2013.

[5] M. Beister, D. Kolditz, and W. A. Kalender, "Iterative reconstruction methods in X-ray CT,” Phys. Medica, vol. 28, no. 2, pp. 94-108, Apr. 2012
[6] C. Mora, M. J. Rodríguez-Álvarez, and J. V. Romero, "New pixellation scheme for CT algebraic reconstruction to exploit matrix symmetries," Comput. Math. with Appl., vol. 56, no. 3, pp. 715-726, Aug. 2008.

[7] J. Kim, D. Ionascu, and T. Zhang, "Technical Note: Algebraic iterative image reconstruction using a cylindrical image grid for tetrahedron beam computed tomography,” Med. Phys., vol. 40, no. 8, p. 81909, Jul. 2013.

[8] M.-J. Rodríguez-Alvarez, F. Sánchez, A. Soriano, A. Iborra, and C. Mora, "Exploiting symmetries for weight matrix design in CT imaging,” Math. Comput. Model., vol. 54, no. 7-8, pp. 1655-1664, Oct. 2011.

[9] M. J. Rodríguez-Alvarez et al., "Expectation maximization (EM) algorithms using polar symmetries for computed tomography (CT) image reconstruction,” Comput. Biol. Med., vol. 43, no. 8, pp. 10531061, Sep. 2013.

[10] W. Yang, X. Xu, K. Bi, S. Zeng, Q. Liu, and S. Chen, “Adaptive region of interest method for analytical micro-CT reconstruction,” J. Xray. Sci. Technol., vol. 19, no. 1, pp. 23-33, 2010.

[11] A. Andersen, "Simultaneous Algebraic Reconstruction Technique (SART): A superior implementation of the ART algorithm," Ultrason. Imaging, vol. 6, no. 1, pp. 81-94, Jan. 1984. 
[12] L. Flores, V. Vidal, E. Parcero, and G. Verdu, "Application of a modified LSQR method for CT imaging reconstruction with low doses to patient,” in 2016 9th International Congress on Image and Signal Processing, BioMedical Engineering and Informatics (CISP-BMEI), 2016, pp. 1969-1974.

[13] A. Beck and M. Teboulle, "A Fast Iterative Shrinkage-Thresholding Algorithm for Linear Inverse Problems,” SIAM J. Imaging Sci., vol. 2, no. 1, pp. 183-202, Jan. 2009.

[14] W. Yu, C. Wang, X. Nie, M. Huang, and L. Wu, "Image Reconstruction for Few-view Computed Tomography Based on $\ell 0$ Sparse Regularization,” Procedia Comput. Sci., vol. 107, pp. 808-813, 2017.

[15] X. Pan, E. Y. Sidky, and M. Vannier, "Why do commercial CT scanners still employ traditional, filtered back-projection for image reconstruction?,” Inverse Probl., vol. 25, no. 12, p. 123009, Dec. 2009.

[16] V. V. Selivanov and R. Lecomte, "Fast PET image reconstruction based on SVD decomposition of the system matrix," IEEE Trans. Nucl. Sci., vol. 48, no. 3, pp. 761-767, Jun. 2001.

[17] Xis han Sun, Tianyu Ma, and Yongjie Jin, "SVD Reconstruction Algorithm in 3D SPECT Imaging," in IEEE Symposium Conference Record Nuclear Science 2004., vol. 6, pp. 3527-3530.

[18] M.-J. Rodríguez-Alvarez, F. Sánchez, A. Soriano, and A. Iborra, "Sparse Givens resolution of large system of linear equations: Applications to image reconstruction,” Math. Comput. Model., vol. 52, no. 7-8, pp. 1258-1264, Oct. 2010.

[19] S. Gou et al., "CT Image Sequence Restoration Based on Sparse and Low-Rank Decomposition,” PLoS One, vol. 8, no. 9, p. e72696, Sep. 2013.

[20] Shewen Sun, S. Wei, and Cong Wang, "DPCC and QR factorizationbased color medical image authentication algorithm,” in 2009 International Conference on Image Analysis and Signal Processing, 2009, pp. 81-84.

[21] W. Qiu, D. Titley-Peloquin, and M. Soleimani, "Blockwise conjugate gradient methods for image reconstruction in volumetric CT," Comput. Methods Programs Biomed, vol. 108, no. 2, pp. 669-678, Nov. 2012. X. Yang, R. Hofmann,

[22] R. Dapp, T. van de Kamp, T. dos Santos Rolo, X. Xiao, J. Moosmann, J. Kashef, R. Stotzka, TV-based conjugate gradient method and discrete L-curve for few-view CT reconstruction of X-ray in vivo data, Opt. Express. 23 (2015) 5368-5387. doi:10.1364/OE.23.005368.

[23] Rit, M. Vila Oliva, S. Brousmiche, R. Labarbe, D. Sarrut, and G. C. Sharp, "The Reconstruction Toolkit (RTK), an open-source cone-beam CT reconstruction toolkit based on the Insight Toolkit (ITK)," J. Phys. Conf. Ser., vol. 489, p. 12079, Mar. 2014.

[24] A. Iborra, et al., "Effect of noise in CT image reconstruction using QRDecomposition algorithm," in 2013 IEEE Nuclear Science Symposium and Medical Imaging Conference (2013 NSS/MIC), 2013, pp. 1-4.

[25] A. Iborra Carreres, "Development of a New 3D Reconstruction Algorithm for Computed Tomography (CT)," Universitat Politècnica de València, Valencia (Spain), 2015. doi:10.4995/Thesis/10251/59421.

[26] G. H. Golub and C. F. Van Loan, Matrix Computations, 4th ed. Baltimore: The Johns Hopkins University Press, 2013.

[27] D. S. Watkins, Fundamentals of Matrix Computations, Third ed. New York: Wiley-Interscience Publication, 2004

[28] F. R. Verdun et al., "Image quality in CT: From physical measurements to model observers," Phys. Medica, vol. 31, no. 8, pp. 823-843, Dec. 2015.

[29] A. Soriano et al., "EM tomographic image reconstruction using polar voxels,” J. Instrum., vol. 8, no. 1, pp. C01004-C01004, Jan. 2013.

[30] A. Iborra et al., "Noise Analysis in Computed Tomography (CT) Image Reconstruction using QR-Decomposition Algorithm," IEEE Trans. Nucl. Sci., vol. 62, no. 3, pp. 869-875, Jun. 2015

[31] K. L. Boedeker, V. N. Cooper, and M. F. McNitt-Gray, "Application of the noise power spectrum in modern diagnostic MDCT: part I. Measurement of noise power spectra and noise equivalent quanta," Phys. Med. Biol., vol. 52, no. 14, pp. 4027-4046, Jul. 2007.

[32] G. Sobel, I., Feldman, "A 3x3 isotropic gradient operator for image processing." in Unpublished, presented as a talk within Stanford Artificial Intelligence Project., 1968.

[33] F. Sánchez et al., "ALBIRA: A small animal PET/SPECT/CT imaging system,” Med. Phys., vol. 40, no. 5, p. 51906, Apr. 2013.

[34] L. Flores, V. Vidal, and G. Verdú, "System Matrix Analysis for Computed Tomography Imaging," PLoS One, vol. 10, no. 11, p. e0143202, Nov. 2015.
[35] W. Yao and K. Leszczynski, "Analytically derived weighting factors for transmission tomography cone beam projections,” Phys. Med. Biol., vol. 54, no. 3, pp. 513-533, Feb. 2009.

[36] J. H. Wilkinson The algebraic eigenvalue problem. Claredom Press, Oxford University, 1965.

[37] N.J. Higham. Accuracy and Stability of numerical algorithms. SIAM, Philadelphia, 1996

[38] E.Y. Sidky, X. Pan, Image reconstruction in circular cone-beam computed tomography by constrained, total-variation minimization, Phys. Med. Biol. 53 (2008) 4777-4807.

[39] J. H. Siewerdsen, I. A. Cunningham, and D. A. Jaffray, "A framework for noise-power spectrum analysis of multidimensional images," Med. Phys., vol. 29, no. 11, pp. 2655-2671, Oct. 2002.

[40] A. Iborra et al., "A new method for image reconstruction in computed tomography (CT) using QR-Decomposition: Image quality assessment," in 2015 IEEE Nuclear Science Symposium and Medical Imaging Conference (NSS/MIC), 2015, pp. 1-4.

[41] S. J. Erasmus and K. C. A. Smith, "An automatic focusing and astigmatism correction system for the SEM and CTEM," J. Microsc., vol. 127, no. 2, pp. 185-199, Aug. 1982.

[42] Zhang, Nien Fan, A. E. Vladar, M. T. Postek, and R. D. Larrabee, “A Kurtosis-Based Statistical Measure for Two-Dimensional Processes and Its Applications to Image Sharpness,” in Section of Physical and Engineering Sciences of American Statistical Society, 2003, pp. 47304736.

[43] J. Caviedes and F. Oberti, "A new sharpness metric based on local kurtosis, edge and energy information," Signal Process. Image Commun., vol. 19, no. 2, pp. 147-161, Feb. 2004.

[44] R. Ferzli and L. J. Karam, "A No-Reference Objective Image Sharpness Metric Based on the Notion of Just Noticeable Blur (JNB)," IEEE Trans. Image Process., vol. 18, no. 4, pp. 717-728, Apr. 2009.

[45] P. Marziliano, F. Dufaux, S. Winkler, and T. Ebrahimi, "A no-reference perceptual blur metric,” in Proceedings. International Conference on Image Processing, vol. 1, p. III-57-III-60. 